TRANSACTIONS OF THE

AMERICAN MATHEMATICAL SOCIETY

Volume 352, Number 10, Pages 4677-4692

S 0002-9947(00)02595-2

Article electronically published on June 14, 2000

\title{
POLYNOMIALS THAT ARE POSITIVE ON AN INTERVAL
}

\author{
VICTORIA POWERS AND BRUCE REZNICK
}

\begin{abstract}
This paper discusses representations of polynomials that are positive on intervals of the real line. An elementary and constructive proof of the following is given: If $h(x), p(x) \in \mathbb{R}[x]$ such that $\{\alpha \in \mathbb{R} \mid h(\alpha) \geq 0\}=[-1,1]$ and $p(x)>0$ on $[-1,1]$, then there exist sums of squares $s(x), t(x) \in \mathbb{R}[x]$ such that $p(x)=s(x)+t(x) h(x)$. Explicit degree bounds for $s$ and $t$ are given, in terms of the degrees of $p$ and $h$ and the location of the roots of $p$. This is a special case of Schmüdgen's Theorem, and extends classical results on representations of polynomials positive on a compact interval. Polynomials positive on the non-compact interval $[0, \infty)$ are also considered.
\end{abstract}

\section{INTRODUCTION}

Suppose that $p \in \mathbb{R}[x]$ is a real polynomial in a single real variable. If $p(x) \geq 0$ for all $x \in \mathbb{R}$, then an easy consequence of the Fundamental Theorem of Algebra is that $p$ can be written as a sum of two squares of polynomials. It is natural to wonder what one can say if $p(x) \geq 0$ or $p(x)>0$ for $x$ in a fixed interval.

There are several such representations which resonate with more recent work in real algebraic geometry. It has long been known that if $p(x)>0$ for $x \in(-1,1)$, then $p$ can be written as a positive linear combination of polynomials $(1-x)^{i}(1+x)^{j}$ for suitable integers $i$ and $j$ (Bernstein); however, it might be necessary for $i+j$ to exceed the degree of $p$. And if $p(x) \geq 0$ for $x \in[-1,1]$, then one can write $p(x)=f(x)+\left(1-x^{2}\right) g(x)$, where $f(x), g(x) \geq 0$ for all $x$ (Fekete). It has also long been known that if $p(x) \geq 0$ for $x \in[0, \infty)$, then $p$ can be written in the form $f_{1}+x f_{2}$, where each $f_{i}$ is a sum of two squares (Pólya-Szegö). The proofs of these results are elementary, and are included in this paper.

This question can be viewed from a more abstract algebraic perspective. Recently, K. Schmüdgen [23] has proved a remarkable theorem which can be viewed as a broad generalization of these representations to positive functions. Roughly speaking, if a compact set $S$ in $\mathbb{R}^{m}$ is defined by finitely many polynomial inequalities, then any polynomial which is strictly positive on $S$ can be written in terms of the defining polynomials for $S$ and sums of squares (of polynomials). The proof of this result in [23] is neither elementary nor constructive.

We now present the requisite definitions. Given non-constant polynomials $f_{1}, \ldots$, $f_{n} \in \mathbb{R}\left[x_{1}, \ldots, x_{m}\right]$, define $S\left(f_{1}, \ldots, f_{n}\right)$ to be the basic closed semi-algebraic set generated by the $f_{i}$ 's, i.e.,

$$
S\left(f_{1}, \ldots, f_{n}\right)=\left\{\alpha \in \mathbb{R}^{m} \mid f_{i}(\alpha) \geq 0 \text { for } 1 \leq i \leq n\right\} .
$$

Received by the editors January 14, 1999.

1991 Mathematics Subject Classification. Primary 14Q20; Secondary 26C99, 68W30.

The second author was supported in part by NSF Grant DMS 95-00507. 
Let $\Sigma$ denote the set of sums of squares $\sum_{j} f_{j}^{2}$, with $f_{j} \in \mathbb{R}\left[x_{1}, \ldots, x_{m}\right]$. For any $I \subset\{1, \ldots, n\}$, let $f_{I}(x)=\prod_{i \in I} f_{i}(x)$, with the usual understanding that $f_{\emptyset}(x)=1$. Then we define $P\left(f_{1}, \ldots, f_{n}\right)$, the preorder generated by the $f_{i}$ 's, by

$$
P\left(f_{1}, \ldots, f_{n}\right)=\left\{\sum_{I \subset\{1, \ldots, n\}} s_{I}(x) f_{I}(x) \mid s_{I} \in \Sigma\right\} .
$$

Note that since $\Sigma$ is closed under multiplication and $f_{I} f_{I^{\prime}}=f_{I \cap I^{\prime}}^{2} f_{I \triangle I^{\prime}}$, the preorder $P\left(f_{1}, \ldots, f_{n}\right)$ is also closed under multiplication.

For a set $A \subseteq \mathbb{R}^{m}$, let $\operatorname{Psd}(A)$ (resp. $\operatorname{Pd}(A)$ ) denote the set of polynomials $p \in \mathbb{R}\left[x_{1}, \ldots, x_{m}\right]$ so that $p\left(a_{1}, \ldots, a_{m}\right) \geq 0$ (resp. $p\left(a_{1}, \ldots, a_{m}\right)>0$ ) for every $\left(a_{1}, \ldots, a_{m}\right) \in A$. Such polynomials are said to be positive semi-definite (psd) on $A$ (resp. positive definite $(p d)$ on $A$ ). These sets are also closed under multiplication.

Let $P=P\left(f_{1}, \ldots, f_{n}\right)$ and $S=S\left(f_{1}, \ldots, f_{n}\right)$, then clearly, $P$ is contained in $\operatorname{Psd}(S)$. Schmüdgen's Theorem says that if $S$ is compact, then a stronger statement is true:

$$
\operatorname{Pd}(S) \subseteq P \subseteq \operatorname{Psd}(S)
$$

In other words, if $g>0$ on compact $S\left(f_{1}, \ldots, f_{n}\right)$, then $g$ is in the preorder generated by the $f_{i}$ 's. Schmüdgen's Theorem is somewhat simpler in one variable. As noted earlier, $f \in \Sigma$ iff $f \in \operatorname{Psd}(\mathbb{R})$; that is, iff $S(f)=\mathbb{R}$. (The situation is more complicated for polynomials in more than one variable; see [20].) Thus, to give a simple example, one consequence of Schmüdgen's Theorem is that if $p(x)>0$ for $x \in[-1,1]$, then one can write $p(x)=f(x)+\left(1-x^{2}\right)^{3} g(x)$, where $f(x), g(x) \geq 0$ for all $x$. This paper contains a constructive proof of this result, with degree bounds for $f$ and $g$ which depend on the degree of $p$ and the location of its roots.

In Section 2 of this paper, we show that the study of $\operatorname{Psd}(I)$ and $\operatorname{Pd}(I)$ for real intervals $I$ essentially reduces to two cases: $I=[-1,1]$ and $I=[0, \infty)$. We review the literature on this problem, which goes back to Hermite, and discuss work of Goursat, Bernstein, Hausdorff, Pòlya and Szegö, Fekete, Lukàcs, Karlin and Shapley and Karlin and Studden. (There has been some work on $\operatorname{Psd}(A)$ when $A \subset \mathbb{R}^{m}$ is given in the form $A=S\left(f_{1}, \ldots, f_{n}\right)$ for linear $f_{i}$. See papers by Handelman [8] and Micchelli and Pinkus [16]. These are beyond the scope of this paper.)

In Section 3. we combine recent results of de Loera and Santos with work of Goursat, Pòlya and Szegö to give a constructive proof that, if $p$ is in $\operatorname{Pd}([-1,1])$, then for a computable value of $m$, there exist $d_{k} \geq 0$ so that

$$
p(x)=\sum_{k=0}^{m} d_{k}(1-x)^{k}(1+x)^{m-k}
$$

(Without the information on $m$, this theorem is due to Bernstein.) Other computations of $m$ have been made by Erdélyi and Erdélyi and Szabados.

In Section 4, we give an elementary and constructive proof of Schmüdgen's Theorem in one special case. Let $h$ be a given polynomial for which $S(h)=[-1,1]$. If $p \in \operatorname{Pd}([-1,1])$, we give a constructive proof of the existence of $s_{0}, s_{1} \in \Sigma$ so that $p=s_{0}+h s_{1}$. This includes an a priori bound on the degrees of $s_{0}$ and $s_{1}$, based on $h$, the degree of $p$, and the smallest absolute value of the roots of $p$. In the special case $h(x)=1-x^{2}$, Lukács proved a stronger theorem: $\operatorname{Psd}([-1,1])=P\left(1-x^{2}\right)$. We 
shall give a necessary and sufficient condition on $h$ so that $\operatorname{Psd}([-1,1])=P(h)$. Our proof relies on a non-constructive result of Scheiderer, and so is not constructive.

In Section [5, we turn our attention to the non-compact interval $[0, \infty)$, to which Schmüdgen's Theorem does not apply. As noted earlier, Pòlya and Szegö proved that, if $p \in \operatorname{Psd}([0, \infty))$, then there exist $s_{i} \in \Sigma$ so that $p(x)=s_{0}(x)+x s_{1}(x)$. In this section, we prove that this is essentially the only case in which Schmüdgen's conclusion holds for $[0, \infty)$ : If $S\left(f_{1}, \ldots, f_{n}\right)=[0, \infty)$ and $\operatorname{Pd}([0, \infty)) \subset P\left(f_{1}, \ldots, f_{n}\right)$, then $f_{j}(x)=c x$ for some $j$.

The final version of this paper was hammered out in October 1998, while the authors were participating in the MSRI Workshop on Symbolic Computation in Algebra, Geometry, and Analysis. We happily acknowledge our gratitude to MSRI for its warm hospitality.

\section{BACKGROUND AND HISTORICAL REMARKS}

Suppose $I \subseteq \mathbb{R}$ is an interval. How can one describe $\operatorname{Psd}(I)$ and $\operatorname{Pd}(I)$ ? At first glance, this question might seem to involve many cases, depending on whether $I$ is open or half-open or closed; finite, half-infinite or infinite. In fact, there are really only two cases.

First observe that, by continuity, $\operatorname{Psd}(I)=\operatorname{Psd}(\bar{I}) ; \operatorname{Pd}(I)$ contains $\operatorname{Pd}(\bar{I})$, with the complement consisting of those polynomials which are positive on $I$ but vanish at one or the other of its endpoints. If $a \in \bar{I} \backslash I$ is a left-hand endpoint and $p \in \operatorname{Pd}(I) \backslash \operatorname{Pd}(\bar{I})$, then there exists an integer $k$ so that $p(x)=(x-a)^{k} q(x)$, where $q \in \operatorname{Pd}(I \cup\{a\})$. A similar consideration applies if $b \in \bar{I} \backslash I$ is a right-hand endpoint. Thus, it suffices to consider $\operatorname{Psd}(I)$ and $\operatorname{Pd}(I)$ for closed intervals $I$. Furthermore, if $p \in \operatorname{Psd}(I) \backslash \operatorname{Pd}(I)$, then $p$ has (finitely many) zeroes in $I$. If $p(t)=0$ and $t$ is interior to $I$, then $p(x)=(x-t)^{2 k} q(t)$ for some even integer $2 k$, and $q \in \operatorname{Psd}(I)$. If $a$ (resp. $b$ ) is a left-hand (resp. right-hand) endpoint of $I$ and $p(a)=0$ (resp. $p(b)=0)$, then $p(x)=(x-a)^{k} q(x)\left(\right.$ resp. $\left.p(x)=(b-x)^{k} q(x)\right)$ for some $q \in \operatorname{Psd}(I)$. In any event, $p$ can only have a finite number of zeroes, so $p \in \operatorname{Psd}(I)$ for the closed interval $I=[a, b]$ if and only if

$$
p(x)=(x-a)^{k_{0}}\left(\prod_{j=1}^{r}\left(x-t_{j}\right)^{2 k_{j}}\right)(b-x)^{k_{r+1}} q(x)
$$

for some non-negative integers $k_{j}$, where $t_{j} \in(a, b)$ and $q \in \operatorname{Pd}(I)$. (If $I$ is halfinfinite or infinite, then this formula is modified accordingly.)

If $I=(-\infty, \infty)$, then it is classically known that $\operatorname{Psd}(I)$ consists of the sums of two squares of polynomials and $\operatorname{Pd}(I)$ consists of the sums of two squares of polynomials which have no common real zeros. If $I$ is half-infinite, then $I=[a, \infty)$ (resp. $(-\infty, b])$, and if $p \in \operatorname{Pd}(I)$ and $f(x)=p(x-a)$ (resp. $f(x)=p(b-x)$ ), then $f \in \operatorname{Pd}([0, \infty))$. Finally, if $I=[a, b], p \in \operatorname{Pd}(I)$ and

$$
f(x)=p\left(\frac{(b-a) x+(b+a)}{2}\right),
$$

then $f \in \operatorname{Pd}([-1,1])$. In other words, there are essentially only two cases: $I=$ $[0, \infty)$ and $I=[-1,1]$.

(It might seem more natural to identify $[0,1]$ as the archetypal finite interval, and the early theorems of Bernstein and Hausdorff were cast in this way. The interval $[-1,1]$ was preferred by analysts wanting to know which polynomials $p$ have the property that the trigonometric polynomial $p(\cos \theta)$ takes non-negative values.) 
It turns out that $\operatorname{Pd}([0, \infty))$ and $\operatorname{Pd}([-1,1])$ are closely related to each other. Given a polynomial $f$ of exact degree $m$, we define the ( $m$-th degree) Goursat transform of $f, \tilde{f}$, by

$$
\tilde{f}(x)=(1+x)^{m} f\left(\frac{1-x}{1+x}\right) .
$$

It is worth noting that the Goursat tranform is nearly its own inverse:

$$
\tilde{\tilde{f}}(x)=(1+x)^{m} \tilde{f}\left(\frac{1-x}{1+x}\right)=(1+x)^{m}\left(1+\frac{1-x}{1+x}\right)^{m} f\left(\frac{1-\frac{1-x}{1+x}}{1+\frac{1-x}{1+x}}\right)=2^{m} f(x) .
$$

If $\operatorname{deg} f=m$ and $\operatorname{deg} \tilde{f}=m-k<m$, then the $(m-k)$-th degree Goursat transform of $\tilde{f}$ is already a polynomial, hence (4) implies that $(1+x)^{k} \mid f(x)$.

Lemma 1 (Goursat's Lemma). Suppose $f$ is a polynomial of degree $m$. Then $f \in$ $\operatorname{Pd}([-1,1])$ if and only if $\tilde{f} \in \operatorname{Pd}([0, \infty))$ and $\operatorname{deg} \tilde{f}=m ; f \in \operatorname{Psd}([-1,1])$ if and only if $\tilde{f} \in \operatorname{Psd}([0, \infty))$ and $\operatorname{deg} \tilde{f} \leq m$.

Proof. If $x \in(-1,1]$, then $y=\frac{1-x}{1+x} \in[0, \infty)$. Since $(1+x)^{m}>0$, we see from (3) that $f(x)>0$ (resp. $f(x) \geq 0$ ) if and only if $\tilde{f}(y)>0$ (resp. $\tilde{f}(y) \geq 0$.) Write $f(x)=\sum_{k=0}^{m} a_{k} x^{k}$; the coefficient of $x^{m}$ in $\tilde{f}(x)=\sum_{k=0}^{m} a_{k}(1-x)^{k}(1+x)^{m-k}$ is $\sum_{k=0}^{m}(-1)^{k} a_{k}=f(-1)$.

It follows immediately that $f \in \operatorname{Pd}([-1,1])$ if and only if $\tilde{f} \in \operatorname{Pd}([0, \infty))$ and $\operatorname{deg} \tilde{f}=m$. If we weaken the hypothesis from "positive" to "non-negative", then the same conclusions carry over, with the loss of information about the degree of $\tilde{f}$. (Note that $f(-1) \geq 0$ by continuity, in any case.)

This subject appears to have been inaugurated [11] in 1894 by the 71-year old French mathematician Charles Hermite, in the first volume of the French problems journal Interméd. des math. Let

$$
\mathcal{P}_{d}:=\left\{\sum_{i+j \leq d} c_{i j}(1-x)^{i}(1+x)^{j} \mid c_{i j} \geq 0\right\} .
$$

Hermite asked: if $p \in \operatorname{Pd}([-1,1])$ has degree $d$, must it belong to $\mathcal{P}_{d}$ ? This question was quickly answered in the negative, by E. Goursat [7], J. Sadier [21] and J. Franel [6], in several different ways. A later solution appeared in Pólya-Szegö [18, VI 48]. We present Goursat's proof.

Suppose $p \in \mathcal{P}_{d}$ and $p(x)=\sum_{i+j \leq d} c_{i j}(1-x)^{i}(1+x)^{j}$, with $c_{i j} \geq 0$. Then

$$
\tilde{p}(x)=\sum_{i+j \leq d} 2^{i+j} c_{i j} x^{i}(1+x)^{d-i-j},
$$

so that the coefficients of $\tilde{p}$ are nonnegative. For $\epsilon>0$, let $p_{\epsilon}(x)=x^{2}+\epsilon$. We have

$$
\tilde{p}_{\epsilon}(x)=\epsilon(1+x)^{2}+(1-x)^{2}=(1+\epsilon)-(2-2 \epsilon) x+(1+\epsilon) x^{2} .
$$

Clearly, if $\epsilon>0$, then $p_{\epsilon} \in \operatorname{Pd}([-1,1])$. But $p_{\epsilon} \in \mathcal{P}_{2}$ if and only if the coefficients of $\tilde{p}_{\epsilon}$ are nonnegative, and this is true only for $\epsilon \geq 1$. Thus, for $0<\epsilon<1, p_{\epsilon}$ provides a negative answer to Hermite's question.

However, if $p \in \operatorname{Pd}([-1,1])$, then it is true that $p \in \mathcal{P}_{m}$ for sufficiently large $m$. This was proved by Bernstein [1] in 1915, although Pólya-Szegö attributes this result to Hausdorff [10, pp. 98-99] in 1921, as part of his solution of the classical moment problem on $[0,1]$. Two proofs of this theorem are given in [18]. One 
uses Goursat's transform, combined with another theorem of Pólya's [17]. We will give a computational version of the latter proof in the next section. Finally, it is worth noting that if $0 \neq p \in \operatorname{Psd}([-1,1])$, and $p(u)=0$ for $u \in(-1,1)$, then upon setting $x=u$ in the equation $p(x)=\sum_{i, j} c_{i j}(1-x)^{i}(1+x)^{j}$, with $c_{i j} \geq 0$, we conclude that $c_{i j}=0$ for all $(i, j)$, a contradiction. It then follows easily that $\bigcup_{m} \mathcal{P}_{m}=\operatorname{Pd}((-1,1))$.

Two other results found in 18 give degree information absent in Schmüdgen's Theorem. If $p(x) \in \operatorname{Psd}([-1,1])$ and $p$ has degree $n$, then

$$
p(x)=(f(x))^{2}+\left(1-x^{2}\right)(g(x))^{2}
$$

for some polynomials $f$ and $g$ of degree at most $n$ and $n-1$ respectively. (This result ([18, VI 46]) is attributed to M. Fekete, but no additional bibliographic details are given.) Under the same hypotheses, $p$ can be written as

$$
p(x)=\left(f_{1}(x)\right)^{2}+(1-x)\left(f_{2}(x)\right)^{2}+(1+x)\left(f_{3}(x)\right)^{2}+\left(1-x^{2}\right)\left(f_{4}(x)\right)^{2}
$$

so that each summand has degree $\leq n$. (This result ([18, VI 47]) is attributed to F. Lukàcs, again with no details.) Further, if $n$ is even, then $f_{2}=f_{3}=0$, and if $n$ is odd, then $f_{1}=f_{4}=0$.

Karlin and Shapley [12, p. 35] gave an even more precise representation. Suppose $p(x) \in \operatorname{Pd}([-1,1])$ has even degree $n=2 m$, then

$$
p(x)=\alpha \prod_{j=1}^{m}\left(x-x_{2 j-1}\right)^{2}+\beta\left(1-x^{2}\right) \prod_{j=1}^{m-1}\left(x-x_{2 j}\right)^{2} .
$$

If $p$ has odd degree $n=2 m+1$, then

$$
p(x)=\alpha(1+x) \prod_{j=1}^{m}\left(x-x_{2 j}\right)^{2}+\beta(1-x) \prod_{j=1}^{m-1}\left(x-x_{2 j-1}\right)^{2} .
$$

These representations are unique under the additional condition that $-1<x_{1}<$ $\cdots<x_{n-1}<1$. Karlin and Studden [13, p. 169] give a similarly interlaced representation for polynomials in $\operatorname{Pd}([0, \infty))$.

We present now a short proof of the representation result [18, VI 45] for $[0, \infty)$.

Proposition 2 (Pólya-Szegö). If $p \in P s d([0, \infty))$, then $p \in P(x)$. More specifically, there exist $f, g \in \Sigma$ so that $p(x)=f(x)+x g(x)$, and $\operatorname{deg} f$, $\operatorname{deg} x g \leq \operatorname{deg} p$.

Proof. First observe that if $p_{i}=f_{i}+x g_{i}$, with $\operatorname{deg} f_{i}, \operatorname{deg} x g_{i} \leq \operatorname{deg} p_{i}$, then

$$
p:=p_{1} p_{2}=\left(f_{1} f_{2}+x^{2} g_{1} g_{2}\right)+x\left(f_{1} g_{2}+f_{2} g_{1}\right):=f+x g,
$$

where $\operatorname{deg} f, \operatorname{deg} x g \leq \operatorname{deg} p$. Thus, it suffices to write $p \in \operatorname{Psd}([0, \infty))$ as a product of factors, each of which satisfies the desired condition.

Now factor $p$ over $\mathbb{R}[x]$. Any positive roots appear to an even degree, hence the linear factors of $p$ will either appear to an even degree, or will be a product of terms $x+x_{0}$, with $x_{0} \geq 0$. The irreducible quadratic factors of $p$ are positive definite. Since any psd factor $q$ is already in $\Sigma$, it can be written as $q+x \cdot 0$; the linear factor $x+x_{0}$ can be written as $x_{0}+x \cdot 1$. In view of the first paragraph, this completes the proof.

Proposition 2 implies a stronger conclusion than Schmüdgen's Theorem for $P\left(1-x^{2}\right)$ and $P(1-x, 1+x)$.

Corollary 3. $\operatorname{Psd}([-1,1])=P\left(1-x^{2}\right)=P(1-x, 1+x)$. 
Proof. Recall that if $p \in P\left(1-x^{2}\right)$, then $p(x)=f(x)+\left(1-x^{2}\right) g(x)$ for $f, g \in \Sigma=$ $\operatorname{Psd}(\mathbb{R})$, so $p \in \operatorname{Psd}([-1,1])$. A similar argument applies to $P(1-x, 1+x)$.

To prove the converse, suppose $p \in \operatorname{Psd}([-1,1])$ and $\operatorname{deg} p=m$. By Goursat's Lemma and Proposition [2, there exist $f_{i}, g_{i} \in \mathbb{R}[x], r_{i}=\operatorname{deg} f_{i} \leq m / 2$ and $s_{i}=$ $\operatorname{deg} g_{i} \leq m / 2$ so that

$$
\tilde{p}(x)=\sum_{i=1}^{2} f_{i}^{2}(x)+x \sum_{i=1}^{2} g_{i}^{2}(x) .
$$

Now perform another Goursat transform of degree $m$ (cf. (4)):

$$
2^{m} p(x)=\sum_{i=1}^{2}(1+x)^{m-2 r_{i}} \tilde{f}_{i}^{2}(x)+(1-x) \sum_{i=1}^{2}(1+x)^{m-1-2 s_{i}} \tilde{g}_{i}^{2}(x) .
$$

If $m$ is even, we can absorb the extra factors of $1+x$ to obtain

$$
2^{m} p(x)=\sum_{i=1}^{2}\left((1+x)^{m / 2-r_{i}} \tilde{f}_{i}(x)\right)^{2}+\left(1-x^{2}\right) \sum_{i=1}^{2}\left((1+x)^{m / 2-1-s_{i}} \tilde{g}_{i}(x)\right)^{2},
$$

so $p \in P\left(1-x^{2}\right)$. If $m$ is odd, then we obtain a similar expression:

$$
\begin{aligned}
2^{m} p(x)=(1 & +x) \sum_{i=1}^{2}\left((1+x)^{(m-1) / 2-r_{i}} \tilde{f}_{i}(x)\right)^{2} \\
& +(1-x) \sum_{i=1}^{2}\left((1+x)^{(m-1) / 2-s_{i}} \tilde{g}_{i}(x)\right)^{2},
\end{aligned}
$$

hence $p \in P(1-x, 1+x)$.

Finally, observe that $P(f)=P(g)$ if and only if $f \in P(g)$ and $g \in P(f)$. We are done if we can show that $1 \pm x \in P\left(1-x^{2}\right)$ and $1-x^{2} \in P(1-x, 1+x)$. The latter is immediate from $\left(1-x^{2}\right)=(1-x)(1+x)$, and the identity

$$
1 \pm x=\frac{(1 \pm x)^{2}}{2}+\frac{1}{2}\left(1-x^{2}\right) \in P\left(1-x^{2}\right) .
$$

Note that

$$
1-x^{2 k}=\left(1-x^{2}\right)\left(1+x^{2}+\cdots+x^{2 k-2}\right)
$$

and

$$
1-x^{2}=\frac{(k-1)-k x^{2}+x^{2 k}}{k}+\frac{1}{k}\left(1-x^{2 k}\right),
$$

where $(k-1)-k x^{2}+x^{2 k}$ is psd by the arithmetic-geometric inequality. Thus, $P\left(1-x^{2}\right)=P\left(1-x^{2 k}\right)$ for all positive integers $k$. However, Stengle [24] has shown that $1-x^{2} \notin P\left(\left(1-x^{2}\right)^{3}\right)$, so $S(f)=S(g)$ does not imply that $P(f)=P(g)$. (See also Corollary 11 below.) 


\section{Computing the Bernstein Degree}

Suppose $f \in \operatorname{Pd}([-1,1])$ has degree $m$. Define $r(f)$ to be the smallest integer $n$ so that $f \in \mathcal{P}_{n} ; r(f)$ has been called the Bernstein degree of $f$ (by DeVore and Lorentz [3]) and the Lorentz degree of $f$ (by Borwein and Erdélyi [2]). Our first task in this section is to compute $r(f)$.

Pólya proved in 1928 [17, that if $p \in \operatorname{Pd}\left(\mathbb{R}_{+}^{m}\right)$, then for sufficiently large $d$, $\left(1+\sum_{j=1}^{m} x_{j}\right)^{d} p\left(x_{1}, \ldots, x_{m}\right)$ has positive coefficients. In particular, if $g \in$ $\operatorname{Pd}([0, \infty))$, then for sufficiently large $d,(1+x)^{d} g(x)$ has non-negative coefficients. Let $\tilde{r}(g)$ denote the smallest integer $d$ for which $(1+x)^{d} g(x)$ has non-negative coefficients. Recently, De Loera and Santos [14, p. 232] have made an algorithmic analysis of [17; there are several unfortunate typos in [14], but the statement below is correct. We use this to give an upper bound for $\tilde{r}(g)$.

Proposition 4 ([14]). Suppose $q\left(x_{1}, \ldots, x_{m}\right)$ is a real homogeneous polynomial of degree $d$, the coefficients of $q$ are bounded above in absolute value by $L$ and $q(u) \geq$ $\lambda>0$ for $u \in \Delta=\left\{\left(u_{1}, \ldots, u_{m}\right) \mid u_{j} \geq 0, \sum_{j} u_{j}=1\right\}$. If

$$
r \geq \frac{L m d^{2}}{\lambda}+m d
$$

then $\left(\sum_{j} x_{j}\right)^{r} q\left(x_{1}, \ldots, x_{m}\right)$ has positive coefficients.

This proposition has an immediate interpretation for (inhomogeneous) polynomials of one variable:

Corollary 5. Suppose $g(x)=\sum_{j=0}^{d} b_{j} x^{j} \in P d([0, \infty)),\left|b_{j}\right| \leq L$ and

$$
\lambda=\min \left\{\sum_{j=0}^{d} b_{j} t^{j}(1-t)^{d-j} \mid t \in[0,1]\right\} .
$$

Then

$$
\tilde{r}(g) \leq 2 d+\left\lceil\frac{2 d^{2} L}{\lambda}\right\rceil .
$$

Proof. Let $q(x, y)=\sum_{j=0}^{d} b_{j} x^{j} y^{d-j}$, and apply Proposition 4 to $q$, noting that $m=2$. For later reference, observe that $\lambda=\inf \left\{(1-t)^{d} g\left(\frac{t}{1-t}\right) \mid t \in[0,1)\right\}$.

Theorem 6. Suppose $f(x) \in P d([-1,1])$ has degree $n$, and let $\tilde{f}(x)=\sum_{j} e_{j} x^{j}$. Let $\lambda$ denote the minimum of $f(x)$ for $x \in[-1,1]$ and let $L$ denote $\max \left\{\left|e_{j}\right|\right\}$. Then

$$
r(f)=n+\tilde{r}(\tilde{f}) \leq 3 n+\left\lceil\frac{2 n^{2} L}{\lambda}\right\rceil .
$$

Proof. First suppose $m=\tilde{r}(\tilde{f})$, so that

$$
(1+x)^{m+n} f\left(\frac{1-x}{1+x}\right)=(1+x)^{m} \tilde{f}(x)=\sum_{k=0}^{m+n} b_{k} x^{k},
$$

with $b_{k} \geq 0$. Apply the Goursat transform (of degree $m+n$ ) to both sides above, to obtain

$$
2^{m+n} f(x)=\sum_{k=0}^{m+n} b_{k}(1-x)^{k}(1+x)^{m+n-k} .
$$


Thus, $r(f) \leq m+n=\tilde{r}(\tilde{f})+n$. This proof of Bernstein's theorem is in [18].

To prove the converse, we first observe that a representation

$$
f(x)=\sum_{i+j \leq d} c_{i j}(1-x)^{i}(1+x)^{j}
$$

with non-negative $c_{i j}$ can always be homogenized:

$$
\begin{gathered}
f(x)=\sum_{i+j \leq r} c_{i j}(1-x)^{i}(1+x)^{j}\left(\frac{1-x}{2}+\frac{1+x}{2}\right)^{r-(i+j)} \\
=\sum_{i+j \leq r} c_{i j}(1-x)^{i}(1+x)^{j} \frac{1}{2^{r-(i+j)}} \sum_{\ell=0}^{r-i-j}\left({ }^{r-(i+j)}\right)(1-x)^{\ell}(1+x)^{r-(i+j+\ell)} \\
:=\sum_{k=0}^{r} d_{k}(1-x)^{k}(1+x)^{r-k},
\end{gathered}
$$

and if $c_{i j} \geq 0$ for all $i, j$, then $d_{k} \geq 0$ for all $k$ as well. Thus, $r(f)$ is always achieved by a homogeneous representation.

If we have such a representation for $f$ with $d_{k} \geq 0$, then, upon taking the Goursat transform of degree $r \geq n$, we get

$$
(1+x)^{r} f\left(\frac{1-x}{1+x}\right)=(1+x)^{r-n} \tilde{f}(x)=2^{r} \sum_{k=0}^{r} d_{k} x^{k} .
$$

Thus, $\tilde{r}(\tilde{f}) \leq r(f)-n$, and so $r(f)=\tilde{r}(\tilde{f})+n$.

Finally, by Corollary $5, \tilde{r}(\tilde{f})-n \leq\left\lceil\frac{2 L n^{2}}{\lambda}\right\rceil+2 n$, where $\lambda$ is the infimum of $(1-t)^{d} \tilde{f}\left(\frac{t}{1-t}\right)$ for $t \in[0,1)$. However,

$$
(1-t)^{d} \tilde{f}\left(\frac{t}{1-t}\right)=(1-t)^{d}\left(1+\frac{t}{1-t}\right)^{d} f\left(\frac{1-\frac{t}{1-t}}{1+\frac{t}{1-t}}\right)=f(1-2 t),
$$

so that $\lambda$ is precisely the minimum of $f$ on $[-1,1]$.

Remark. Erdélyi [4] and Erdélyi and Szabados [5] have given detailed computations of $r(f)$ for quadratic polynomials. For example, if the (complex) roots of the definite quadratic $f$ lie on the ellipse $x^{2}+\frac{y^{2}}{a^{2}}=1$, then $r(f) \in\left[a^{-2}, 1+2 a^{-2}\right]$, and these bounds are essentially achieved. These results can be found in [2, pp.83-89].

Example. We compute $r\left(p_{\epsilon}\right)$ for $\epsilon>0$, where $p_{\epsilon}(x)=\epsilon+x^{2} \in \operatorname{Pd}([-1,1])$. Recall that

$$
\tilde{p}_{\epsilon}(x)=\epsilon(1+x)^{2}+(1-x)^{2}=(1+\epsilon)-(2-2 \epsilon) x+(1+\epsilon) x^{2},
$$

so that $r\left(p_{\epsilon}\right)=2$ if $\epsilon \geq 1$. Henceforth, assume $\epsilon<1$, and that $\epsilon^{-1} \in(2 k-1,2 k+1]$ for some positive integer $k$. We shall show that $r\left(p_{\epsilon}\right)=2 k+1$, the least odd integer $\geq \epsilon^{-1}$. (For $\epsilon \leq 1 / 3$, compare with Theorem [6. We have $n=2, \lambda=\epsilon$, and $\ell=\max \{1+\epsilon, 2-2 \epsilon\}$, which yields an upper bound of $3 \cdot 2+\left\lceil 2 \cdot 2^{2} \cdot \frac{2-2 \epsilon}{\epsilon}\right\rceil=\left\lceil\frac{16}{\epsilon}\right\rceil-10$.) Let $g_{\delta}(x)=1-(2-\delta) x+x^{2}$, so that $\tilde{p}_{\epsilon}=(1+\epsilon) g_{\delta}$, where $\delta=\frac{4 \epsilon}{1+\epsilon}$. We have $\delta \in\left[\frac{2}{k+1}, \frac{2}{k}\right)$, and $\tilde{r}\left(\tilde{p}_{\epsilon}\right)=\tilde{r}\left(g_{\delta}\right)$.

By the binomial theorem,

$$
(1+x)^{m} g_{\delta}(x)=\sum_{j=0}^{m+2}\left(\left(\begin{array}{c}
m \\
j
\end{array}\right)-(2-\delta)\left(\begin{array}{c}
m \\
j-1
\end{array}\right)+\left(\begin{array}{c}
m \\
j-2
\end{array}\right)\right) x^{j}
$$


POLYNOMIALS THAT ARE POSITIVE ON AN INTERVAL

a further calculation shows that

$$
(1+x)^{m} g_{\delta}(x)=\sum_{j=0}^{m+2} \frac{m !}{j !(m+2-j) !}\left((m+2-2 j)^{2}-(m+2)+j(m+2-j) \delta\right) x^{j} .
$$

Thus $\tilde{r}\left(g_{\delta}\right)$ is the smallest $m$ so that

$$
(m+2-2 j)^{2}-(m+2)+j(m+2-j) \delta \geq 0 \quad \text { for all } 0 \leq j \leq m+2 .
$$

Suppose (71) holds for even $m=2 s$. The inequality for $j=s+1$ implies that $\delta \geq \frac{2}{s+1}$, so $s \geq k$. The algebraic identity

$$
\begin{aligned}
(2 s+ & 2-2 j)^{2}-(2 s+2)+j(2 s+2-j) \delta \\
& =\frac{2(2 s+1)(s+1-j)^{2}}{s+1}+j(2 s+2-j)\left(\delta-\frac{2}{s+1}\right)
\end{aligned}
$$

shows that (17) holds when $\delta \geq \frac{2}{s+1}$. Similarly, if (17) holds for odd $m=2 s-1$, then $j=s$ implies $\delta \geq \frac{2}{s+1}$, so $s \geq k$, and the identity

$$
\begin{aligned}
(2 s+ & 1-2 j)^{2}-(2 s+1)+j(2 s+1-j) \delta \\
& =\frac{2(2 s+1)(s-(j-1))(s-j)}{s+1}+j(2 s+1-j)\left(\delta-\frac{2}{s+1}\right)
\end{aligned}
$$

shows that (7) holds when $\delta \geq \frac{2}{s+1}$, since $j$ is an integer-valued variable.

Since we want to find the smallest $m$ so that (7) holds, for given $\delta$, we conclude that $\tilde{r}\left(g_{\delta}\right)=2 k-1$, and so $r\left(p_{\epsilon}\right)=\tilde{r}\left(\tilde{p}_{\epsilon}\right)+2=\tilde{r}\left(g_{\delta}\right)+2=2 k+1$. The computation in this example is quite similar to [9] pp. 59-60].

\section{SChmüdgen's TheOrem FOR $[-1,1]$}

In this section, we give a constructive proof of Schmüdgen's Theorem in the special case that there is a single polynomial $h$ such that $S(h)=[-1,1]$. That is, if $[-1,1]=\{x: h(x) \geq 0\}$, and $p(x)>0$ for $x \in[-1,1]$, then we construct psd real polynomials $s$ and $t$ so that

$$
p=s+t h \text {. }
$$

(As noted earlier, in one variable $\Sigma$ consists of the psd polynomials, so it suffices to show that $s$ and $t$ are non-negative on $\mathbb{R}$.)

We begin with some simple remarks. First, if $S(h)=[-1,1]$, then $h(x)$ changes sign only at $x= \pm 1$, and can have zeroes with even degree only in $(-1,1)$. Thus $h(x)=(1-x)^{c}(1+x)^{d} q(x)$, where $q( \pm 1) \neq 0$ and $c$ and $d$ are both odd. Furthermore, $q(x) \geq 0$ on $(-1,1)$ and $q(x)>0$ for $|x| \geq 1$. It follows from (1) that, for any polynomial $\phi, P(h) \supset P\left(\phi^{2} h\right)$. Thus, since $c-d$ is even, we can multiply $h$ by an even power of $1 \pm x$ and assume that $c=d=: r$ is odd.

Fix $h(x)=\left(1-x^{2}\right)^{r} q(x)$ so that $S(h)=[-1,1]$. Since $q$ is a polynomial in one variable, $q(x) \nrightarrow 0$ as $x \rightarrow \infty$, so there exists $\alpha$ so that $q(x) \geq \alpha>0$ for $x \in(-\infty, 1] \cup[1, \infty)$. Since $q$ is continuous, there exists $\beta$ so that $0 \leq q(x) \leq \beta$ for $x \in[-1,1]$.

The main technical result is the following:

Theorem 7. Suppose $\epsilon>0$, and $h(x)=\left(1-x^{2}\right)^{r} q(x)$, where $q(x) \geq \alpha>0$ for $x \in(-\infty,-1] \cup[1, \infty)$ and $0 \leq q(x) \leq \beta$ for $x \in[-1,1]$. Let

$$
A=\frac{1}{r 2^{r} \epsilon^{r-1} \alpha}, \quad \text { and } \quad m=\left\lfloor\frac{r}{2 \epsilon}\left(\frac{\beta}{r \alpha}\right)^{\frac{1}{r}}\right\rfloor \text {, }
$$

and set $F(x)=1+\epsilon+x-A x^{2 m} h(x)$. Then $F(x)$ is psd. 
Remark. This theorem implies that $F \in \Sigma$, hence $1+\epsilon+x=F+A x^{2 m} h \in P(h)$. Let $\bar{h}(x)=h(-x)$, then, with the same values for $\alpha, \beta, A$ and $M$, we have $1+\epsilon+x \in$ $P(\bar{h})$; upon taking $x \rightarrow-x$, we see that $1+\epsilon-x \in P(h)$. Thus,

$$
1+\epsilon \pm x=F_{ \pm}(x)+G_{ \pm}(x) h(x),
$$

where $F_{ \pm}, G_{ \pm} \in \Sigma$, and $\operatorname{deg} F_{ \pm}, \operatorname{deg} G_{ \pm} h$ are bounded above by

$$
\left\lfloor\frac{r}{\epsilon}\left(\frac{\beta}{r \alpha}\right)^{\frac{1}{r}}\right\rfloor+\operatorname{deg} h \leq \frac{r}{\epsilon}\left(\frac{\beta}{r \alpha}\right)^{\frac{1}{r}}+\operatorname{deg} h .
$$

Proof. We want to show that $F(x) \geq 0$ for all $x \in \mathbb{R}$. Observe that $1+\epsilon+x \geq 0$ for $x \geq-1-\epsilon$ and $-A x^{2 m} h(x) \geq 0$ for $|x| \geq 1$. Thus we need only consider $x \in(-\infty,-1-\epsilon) \cup(-1,1)$.

First, write $x \in(-\infty,-1-\epsilon)$ as $x=-1-\epsilon-y$, where $y>0$. We must show that

$$
F(x)=-y-A(1+\epsilon+y)^{2 m}\left(1-(1+\epsilon+y)^{2}\right)^{r} q(-1-\epsilon-y) \geq 0 .
$$

We have the estimates $(1+\epsilon+y)^{2 m} \geq 1$ and $q(-1-\epsilon-y) \geq \alpha$, and since $r$ is odd,

$$
\left(1-(1+\epsilon+y)^{2}\right)^{r}=(2+\epsilon+y)^{r}(\epsilon+y)^{r} \geq 2^{r}\left(r \epsilon^{r-1} y\right) .
$$

(The last inequality follows from $\epsilon, y \geq 0$, and the selection of one term from the binomial expansion of $(\epsilon+y)^{r}$.) Thus it suffices to show that

$$
-y+A 2^{r} r \epsilon^{r-1} y \alpha=y\left(A \alpha 2^{r} r \epsilon^{r-1}-1\right) \geq 0,
$$

and this follows directly from the definition of $A$.

Now suppose $x \in(-1,1)$. An easy calculus exercise shows that if $a$ and $b$ are positive and $0 \leq t \leq 1$, then $t^{a}(1-t)^{b} \leq \frac{a^{a} b^{b}}{(a+b)^{a+b}}$. Using this argument with $t=x^{2}$, we have

$$
F(x)=1+\epsilon+x-A\left(x^{2}\right)^{m}\left(1-x^{2}\right)^{r} q(x) \geq \epsilon-A \frac{m^{m} r^{r}}{(m+r)^{m+r}} \beta .
$$

But $\frac{m}{m+r}<1$, and since $m+r>\frac{r}{2 \epsilon}\left(\frac{\beta}{r \alpha}\right)^{1 / r}$, we have

$$
\epsilon-A \frac{m^{m} r^{r}}{(m+r)^{m+r}} \beta>\epsilon-A \beta\left(\frac{r}{m+r}\right)^{r}=\epsilon\left(1-\frac{\beta}{r \alpha}\left(\frac{r}{2 \epsilon(m+r)}\right)^{r}\right) \geq 0 .
$$

Remark. For $A \in \mathbb{R}^{+}$and $m \in \mathbb{N}$, let $F(m, A ; x):=1+\epsilon+x-A x^{2 m} h(x)$. We have not attempted to be precise in the calculation of $m$, and as one might suspect, for particular examples $F(m, A ; x)$ will be psd for a smaller value of $m$ than the one asserted above. Note, for example, that the argument of the last theorem works with the value of $m$ reduced roughly by $r$, provided $\epsilon$ is small enough that this integer is non-negative.

Consider $h(x)=1-x^{2}$, for which, as we've seen, no additional machinery is required to prove Schmüdgen's Theorem. The following identity shows that $m=0$ will actually work: if $a>1$, then $a \pm x=\frac{a}{2}\left(x \pm \frac{1}{a}\right)^{2}+\frac{a^{2}-1}{2 a}+\frac{a}{2}\left(1-x^{2}\right)$. If we take $h(x)=\left(1-x^{2}\right)^{3}$, then $r=3$ and $q(x)=1$, so $\alpha=\beta=1$, and the bound for $m$ is $\frac{3^{2 / 3}}{2 \epsilon}-3 \approx \frac{1.04}{\epsilon}-3$. More precise results can be computed in this specific case. It can be shown, for example, that the minimum of $x-\left(1-x^{2}\right)^{3}$ on $\mathbb{R}$ is approximately -1.11987 ; hence, for example, with $\epsilon=.12, F(0,1 ; x)=1.12+x-\left(1-x^{2}\right)^{3}$ is psd. 
On the other hand, set $\epsilon=.01$ and consider $F(0, A ; x)=1.01+x-A\left(1-x^{2}\right)^{3}$. If $F(0, A ; 0) \geq 0$, then $1.01 \geq A$, but if $F(0, A ;-1.02) \geq 0$, then $A(.0404)^{3} \geq .01$, hence $A>150$. Thus, $F(0, A, x)$ is not psd for any $A$. (This does not imply that $1.01+x \notin P\left(\left(1-x^{2}\right)^{3}\right)$, merely that there is no expression of this simple form.) We can repeat this argument for $m=1$ : $F(1, A ;-.6) \geq 0$ implies $A \leq 4.3445 \ldots$ and $F(1, A ;-.1 .02) \geq 0$ implies $A \geq 145.764 \ldots$. These arguments can be repeated to show that if $1.01+x-A x^{2 m}$ is psd, then $m \geq 11$, and a numerical argument shows that for all $x$,

$$
F(11,140 ; x)=1.01+x-140 x^{22}\left(1-x^{2}\right)^{3} \geq .0002>0 .
$$

The following quantitative special case of Schmüdgen's Theorem contains degree information unavailable in the original. Observe that, if $p$ is positive on $[-1,1]$, then by continuity, it is positive on $[-1-\epsilon, 1+\epsilon]$ for some $\epsilon>0$.

Corollary 8. Suppose $h(x)=\left(1-x^{2}\right)^{r} q(x)$, where $r$ is odd, $0 \leq q(x) \leq \beta$ for $|x| \leq 1$ and $q(x) \geq \alpha>0$ for $|x| \geq 1$. Suppose $p(x) \in P d([-1,1])$ has degree $n$, and $p$ has $s$ real roots $u_{j}$, with $\left|u_{j}\right| \geq u>1$. Then there exist polynomials $F, G \in \Sigma$, so that

$$
p=F+G h,
$$

where the degrees of $F$ and $G$ are bounded above by

$$
s\left(\frac{r}{2(u-1)}\left(\frac{\beta}{\alpha}\right)^{\frac{1}{r}}+\operatorname{deg} h\right)+n-s .
$$

Proof. Factor $p$ over $\mathbb{R}[x]$ into linear factors $x-u_{j}$ and irreducible quadratic factors $q_{k} \in \Sigma$. Then $\left|u_{j}\right| \geq u>1$. Observe that $\pm\left(x-u_{j}\right)=\left|u_{j}\right|-\operatorname{sgn}\left(u_{j}\right) x$, with the sign chosen so that the factor is positive on $[-1,1]$; thus,

$$
p(x)=\prod_{j=1}^{s}\left(\left|u_{j}\right|-\operatorname{sgn}\left(u_{j}\right) x\right) \prod_{k=1}^{\frac{n-s}{2}} q_{k}(x) .
$$

Now use Theorem [6 to write $\left|u_{j}\right|-\operatorname{sgn}\left(u_{j}\right) x=F_{j}+h G_{j}$, with the degrees $\leq$ $\frac{r}{2\left(\left|u_{j}\right|-1\right)}\left(\frac{\beta}{\alpha}\right)^{\frac{1}{r}}+\operatorname{deg} h \leq \frac{r}{2(u-1)}\left(\frac{\beta}{\alpha}\right)^{\frac{1}{r}}+\operatorname{deg} h$, and substitute above.

There is no degree dependence in Fekete's Theorem on the location of the roots of $p$. On the other hand, Stengle has shown [24, p. 170] that there is a constant $C$ such that given $s_{i} \in \Sigma$ with

$$
1-x^{2}+\epsilon=s_{0}(x)+s_{1}(x)\left(1-x^{2}\right)^{3},
$$

then $\operatorname{deg} s_{i} \geq C \epsilon^{-1 / 2}$. Stengle [24, p. 171] also constructed such a representation in which $\operatorname{deg} s_{i} \leq C \epsilon^{-1 / 2}|\log \epsilon|$. This implies that if $r \geq 3$, then there is no bound for the degrees of $F$ and $G$ which depends solely on the degree of $p$ and information about $h$. It also suggests that a better construction might reduce the exponent on $u-1$ from 1 to $\frac{1}{2}$.

Corollary 8 states that $\operatorname{Pd}([-1,1]) \subseteq P(h)$. In view of the results of Fekete and Lukacs, as well as Corollary 3, it is natural to wonder when we have the stronger result that $\operatorname{Psd}([-1,1])=P(h)$. We are able to answer that question completely: this happens if and only if $h(x)=\left(1-x^{2}\right) q(x)$, where $q$ is as before, and $q( \pm 1)>0$. Our proof relies on a very recent result of Scheiderer [22, 4.8]. 
Lemma 9 (Scheiderer). Suppose $f, g \in \mathbb{R}[x]$ satisfy the following conditions:

1. $f$ and $g$ are relatively prime,

2. $\{x \mid f(x) \leq 0\} \cap\{x \mid g(x) \leq 0\}=\emptyset$, and

3. $S(f, g)$ is bounded.

Then there exist $s, t \in \Sigma$ such that $s f+t g=1$.

Proposition 10. Suppose $h \in \mathbb{R}[x]$ such that $S(h)=[-1,1]$ and $h$ has a root of multiplicity $a$ at $x=1$ and a root of multiplicity $b$ at $x=-1$. Then $(1-x)^{a}$ and $(1+x)^{b}$ are in $P(h)$.

Proof. We give the proof for $(1-x)^{a}$; the proof for $(1+x)^{b}$ is similar. Write $h(x)=(1-x)^{a} q(x)$, and note that $a$ must be odd. We want to apply Lemma 9 to the polynomials $(1-x)^{a}$ and $q(x)$. They are clearly relatively prime. We have $\left\{x \mid(1-x)^{a} \leq 0\right\}=[1, \infty)$, and $q(x)>0$ on $[1, \infty)$ by its definition. Hence conditions (2) and (3) of Lemma 9 hold. Thus there exist $s, t \in \Sigma$ so that $1=$ $s(x)(1-x)^{a}+t(x) q(x)$. Multiplying both sides by $(1-x)^{a}$ yields $(1-x)^{a}=$ $s(x)(1-x)^{2 a}+t(x) h(x) \in P(h)$.

Remark. The proof of Lemma 9 is not constructive, hence this does not yield a constructive method for finding an explicit representation of $(1-x)^{a}$ and $(1+x)^{b}$ in the preorder.

Corollary 11. Suppose $S(h)=[-1,1]$ and $p \in \operatorname{Psd}([-1,1])$. Then $p \in P(h)$ if and only if for $y= \pm 1$, the order of $p$ at $y$ is either even or at least as big as the order of $h$ at $y$. In particular, $P(h)=\operatorname{Psd}([-1,1])$ if and only if the order of $h$ at both \pm 1 is 1 .

Proof. Write $h(x)=(1-x)^{a}(1+x)^{b} q(x)$, where $q(x)>0$ for $|x| \geq 1$ and $q(x) \geq 0$ for $|x|<1$. Every $p \in \operatorname{Psd}([-1,1])$ can be written as

$$
p(x)=(1+x)^{c}\left(\prod_{j=1}^{r}\left(x-x_{j}\right)^{2 k_{j}}\right)(1-x)^{d} q(x),
$$

where $\left|x_{j}\right|<1$ and $q \in \operatorname{Pd}([-1,1])$. If $c$ is even or $c-a \geq 0$ is even and if $d$ is even or $d-b \geq 0$ is even, then by Corollary 8 and Proposition $10, p$ is a product of factors from $P(h)$, hence $p \in P(h)$.

Now suppose $c<a$ is odd or $d<b$ is odd. The proofs are similar, and we give the first. Suppose we could write $p=s+t h$, with $s, t \in \Sigma$. Then $(1+x)^{c} \mid p$ and $(1+x)^{a} \mid h$, hence $(1+x)^{c} \mid s$. But $s$ is psd, so any linear factors appear to an even degree. Thus, $(1+x)^{c+1} \mid s$, and $c+1<a$ implies that $(1+x)^{c+1} \mid p$, which contradicts the definition of $c$.

There are several other directions in which these results could be generalized. The most obvious is to allow $\left\{f_{1}, \ldots, f_{n}\right\}$ with $n \geq 2$ so that $S\left(f_{1}, \ldots, f_{n}\right)=[-1,1]$. (As noted earlier, any compact interval might as well be $[-1,1]$, but Schmüdgen's Theorem also applies when the semi-algebraic set is a union of closed intervals.) Stengle [24] proved that if $S\left(f_{1}, \ldots, f_{n}\right)=[-1,1]$, then there exists a single $h$ in $P\left(f_{1}, \ldots, f_{n}\right)$ so that $S(h)=[-1,1]$. His proof requires various non-constructive Stellensätze, and we have been unable to find a constructive proof.

Nevertheless, we can present here some remarks towards a constructive proof. Suppose $S\left(f_{1}, \ldots, f_{n}\right)=[-1,1]$. Assume each $f_{i}$ has a factor of $1-x^{2}$. (If not, replace $f_{i}$ by $\left(1-x^{2}\right)^{2} f_{i} \in P\left(f_{1}, \ldots, f_{n}\right)$, so that $f_{i}=\left(1-x^{2}\right) g_{i}$.) There is no 
real $\alpha$ so that $g_{i}(\alpha)<0$ for all $i$. Our goal is to find psd $p_{1}, \ldots, p_{k}$ such that $p_{1} g_{1}+\cdots+p_{k} g_{k}$ is positive definite. Setting $h=\sum_{k} p_{k} f_{k}=\left(1-x^{2}\right) \sum p_{k} g_{k}$, we then have $S(h)=[-1,1]$, and we can apply Theorem[7.

Example. Suppose $f_{1}=\left(1-x^{2}\right)(x+a)$ and $f_{2}=b^{2}-x^{2}$, where $1<b<a$. Then $S\left(f_{1}, f_{2}\right)=\left\{\alpha: f_{1}(\alpha) \geq 0\right\} \cap\left\{\alpha: f_{2}(\alpha) \geq 0\right\}=((-\infty,-a) \cup[-1,1]) \cap[-b, b]=$ $[-1,1]$. An algebraic identity shows that

$$
\begin{gathered}
h(x)=2 b\left(b^{2}-1\right) f_{1}(x)+\left(1-x^{2}\right)^{2} f_{2}(x) \\
=2 b\left(b^{2}-1\right)\left(1-x^{2}\right)(x+a)+\left(1-x^{2}\right)^{2}\left(b^{2}-x^{2}\right) \\
=\left(1-x^{2}\right)\left(2 b\left(b^{2}-1\right)(x+b+(a-b))+\left(1-x^{2}\right)\left(b^{2}-x^{2}\right)\right) \\
=\left(1-x^{2}\right)\left((x+b)^{2}\left((x-b)^{2}+b^{2}-1\right)+2 b\left(b^{2}-1\right)(a-b)\right) .
\end{gathered}
$$

Since $(x+b)^{2}\left((x-b)^{2}+b^{2}-1\right)+2 b\left(b^{2}-1\right)(a-b) \geq 2 b\left(b^{2}-1\right)(a-b)>0$, we have $S(h)=[-1,1]$. In other words, we have used the above construction with $g_{1}=x+a, g_{2}=\left(1-x^{2}\right)\left(b^{2}-x^{2}\right), p_{1}=2 b\left(b^{2}-1\right)$, and $p_{2}=1$.

It is difficult to apply Theorem 7 directly to $h$ in this case, because of the computation of $\alpha$ and $\beta$. For concreteness, set $a=3$ and $b=2$, so that $2 b\left(b^{2}-1\right)=$ $12, h(x)=\left(1-x^{2}\right)\left(x^{4}-5 x^{2}+12 x+40\right)$ and $r=1$. It is routine to verify that $q(x)=x^{4}-5 x^{2}+12 x+40$ is increasing on $[-1,1]$, hence $\beta=h(1)=48$, and $q(x)=12+(x+2)^{2}\left(x^{2}-4 x+7\right)$, hence $\alpha=12$. If we set $\epsilon=1$, then Theorem 7 tells us that, with $A=\frac{1}{12}$ and $m=1$,

$$
H(x):=2+x-\frac{1}{12} x^{2}\left(1-x^{2}\right)\left(x^{4}-5 x^{2}+12 x+40\right)
$$

is psd. (This can easily be confirmed by graphing it.) That is, we can explicitly write $2+x \in \operatorname{Pd}([-1,1])$ as an element of $P\left(f_{1}, f_{2}\right)$ :

$$
2+x=H(x)+\frac{1}{12} x^{2} h(x)=H(x)+12 x^{2} f_{1}(x)+\frac{1}{12} x^{2}\left(1-x^{2}\right)^{2} f_{2}(x) .
$$

\section{The SEMI-INFInite INTERVAL}

A simple example shows that there exists $h$ so that $S(h)=[0, \infty)$, but $\operatorname{Pd}([0, \infty))$ is not contained in $P(h)$.

Example. Observe that $S\left(x^{3}\right)=[0, \infty)$, and that $1+x \in \operatorname{Pd}([0, \infty))$. But if $1+x \in P\left(x^{3}\right)$, then there would exist $g, h \in \Sigma=\operatorname{Psd}(\mathbb{R})$ so that

$$
1+x=g(x)+x^{3} h(x) .
$$

Observe that the degrees of $g$ and $h$ are even. Hence, the degrees of $g(x)$ and $x^{3} h(x)$, namely, $2 m$ and $2 n+3$, are different, and hence the degree of their sum is $\max \{2 m, 2 n+3\}$, which must equal 1; a clear contradiction.

This example generalizes considerably. We first need a familiar folk-lemma.

Lemma 12. Suppose $-\infty<a<b<\infty$ are given. Then for every positive integer $n$, there exists $C(a, b, n)$ so that, if $\left|\sum_{j=0}^{n} a_{j} x^{j}\right| \leq M$ for $x \in[a, b]$, then $\left|a_{j}\right| \leq$ $M \cdot C(a, b, n)$.

Proof. Let $p(x)=\sum_{j=0}^{n} a_{j} x^{j}$ and choose nodes $a=x_{0}<x_{1}<\cdots<x_{n}=b$. The following system of $n+1$ equations in the $n+1$ unknowns $\left\{a_{j}\right\}$ :

$$
p\left(x_{k}\right)=\sum_{j=0}^{n} x_{k}^{j} a_{j}
$$


has Vandermonde determinant $\prod_{0 \leq j<k \leq n}\left(x_{j}-x_{k}\right) \neq 0$, hence there exist $c_{j, k}$ so that

$$
a_{j}=\sum_{k=0}^{n} c_{j, k} p\left(x_{k}\right)
$$

The assertion is now immediate.

Remark. We note the following useful property of $P\left(f_{1}, \ldots, f_{n}\right)$ in the case where $S\left(f_{1}, \ldots, f_{n}\right)=[0, \infty)$ : Each $f_{i}$ and each $s \in \Sigma$ has positive leading coefficient. Thus, there can be no cancellation in the highest degree terms. In particular, if $p=\sum f_{I} s_{I}$, then for each $I, \operatorname{deg} p \geq \operatorname{deg} s_{I} f_{I}$.

Theorem 13. Suppose $[0, \infty)=S\left(f_{1}, \ldots, f_{n}\right)$ and suppose $\left\{p_{m}\right\} \subset P\left(f_{1}, \ldots, f_{n}\right)$ satisfy $\operatorname{deg} p_{m}=\operatorname{deg} p$ and $p_{m} \rightarrow p$ coefficientwise. Then $p \in P\left(f_{1}, \ldots, f_{n}\right)$.

Proof. Write

$$
p_{m}(x)=s_{0, m}(x)+\sum_{I \subset\{1, \ldots, n\}} s_{I, m}(x) \prod_{i \in I} f_{i}(x),
$$

with $s_{0, m}, s_{I, m} \in \Sigma$, and any summand which is zero has been deleted from the sum. For each $m, \operatorname{deg} p=\operatorname{deg} p_{m}=\max \left\{\operatorname{deg} s_{I, m}+\sum_{i \in I} \operatorname{deg} f_{i}\right\}$, hence there is a uniform bound on the degrees of $\operatorname{deg} s_{I, m}$. Each $f_{i}(x)$ is a polynomial which is non-negative on $[0, \infty)$, hence there exists an interval $\left(0, \lambda_{i}\right)$ on which $f_{i}(x)>0$. Let $\lambda=\min _{i} \lambda_{i}$. Then there exists $\epsilon>0$ so that $f_{i}(x) \geq \epsilon$ for $x \in\left[\frac{\lambda}{3}, \frac{2 \lambda}{3}\right]:=[a, b]$. Since $p_{m} \rightarrow p$, there exists $T$ so that $p_{m}(x) \leq T$ for all $m$ and $x \in[a, b]$. It follows from (9) that, for every subset $I$, and $x \in[a, b]$,

$$
T \geq p_{m}(x) \geq s_{I, m}(x) \prod_{i \in I} f_{i}(x) \geq \epsilon^{|I|} s_{I, m}(x) .
$$

Thus, $s_{I, m}(x) \leq \epsilon^{-|I|} T$ for $x \in[a, b]$, and $\operatorname{deg} s_{I, m} \leq \operatorname{deg} p$. It follows from Lemma 12 that there is a uniform upper bound to the coefficients of $s_{I, m}(x)$. Thus there exists a convergent subsequence $\left\{m_{r}\right\}$ so that, for each $I, s_{I, m_{r}}(x) \rightarrow s_{I}(x)$ coefficientwise. We conclude from (9) that

$$
p(x)=s_{0}(x)+\sum_{I \subset\{1, \ldots, n\}} s_{I}(x) \prod_{i \in I} f_{i}(x) .
$$

Corollary 14. Suppose $S\left(f_{1}, \ldots, f_{n}\right)=[0, \infty)$ and $\epsilon+x \in P\left(f_{1}, \ldots, f_{n}\right)$ for every $\epsilon>0$. Then $f_{j}(x)=$ cx for some $j$ and positive $c$.

Proof. In view of Theorem 13 it suffices to show that $x \in P\left(f_{1}, \ldots, f_{n}\right)$ implies that some $f_{j}$ is a multiple of $x$. Suppose otherwise, and write

$$
x=s_{\emptyset}(x)+\sum_{I \subset\{1, \ldots, n\}} s_{I}(x) \prod_{i \in I} f_{i}(x) .
$$

Each summand has degree $\leq 1$. Since $s_{I} \in \Sigma$, it must be a non-negative constant: $s_{I}(x)=\sigma_{I}$. Further, if $\sigma_{I}>0$, then $\operatorname{deg} \prod_{i \in I} f_{i}(x) \leq 1$, so $I$ is a singleton, $I=\{j\}$, where $f_{j}(x)=a_{j} x+b_{j}$ is linear. Since $f_{j}(x) \geq 0$ on $[0, \infty)$, we have $a_{j}, b_{j} \geq 0$, and $b_{j}>0$ by hypothesis. After a suitable relabeling, we obtain the identity

$$
x=\sigma_{0}+\sum_{j=1}^{r} \sigma_{j}\left(a_{j} x+b_{j}\right) .
$$


By setting $x=0$, we conclude that $\sigma_{0}=\sigma_{1}=\cdots=\sigma_{r}=0$, a contradiction.

Example. Let $p(x)=x+x^{3}$; clearly, $S(p)=[0, \infty)$. We show that, for every odd integer $m$, and $\epsilon$ sufficiently small, $x^{m}+\epsilon \notin P(p)$. It suffices to show by induction on odd $m$ that $x^{m} \notin P(p)$. Suppose otherwise. There would exist $g, h \in \Sigma$ so that

$$
x^{m}=\left(x+x^{3}\right) g(x)+h(x) .
$$

Observe that $m=\max \{3+\operatorname{deg} g, \operatorname{deg} h\}$, where $\operatorname{deg} g, \operatorname{deg} h$ are even. This is impossible for $m=1$. Suppose we have shown this to be impossible for $m-2$, where $m \geq 3$. We have $h(0)=0$, and so $x \mid h(x)$, and since $h \in \Sigma$, it follows that $h(x)=x^{2} \bar{h}(x)$ for some $\bar{h} \in \Sigma$. Thus,

$$
x^{m-1}=\left(1+x^{2}\right) g(x)+x \bar{h}(x) .
$$

But this implies that $g(0)=0$, so $x \mid g(x)$ and so $g(x)=x^{2} \bar{g}(x)$, with $\bar{g} \in \Sigma$, hence

$$
x^{m-2}=\left(x+x^{3}\right) \bar{g}(x)+\bar{h}(x),
$$

which contradicts the induction hypothesis.

Added in proof. There is a gap in the proof of [14, Theorem 1.1]. For details, see 15]. We have been able to improve the bound in [14] as follows:

Theorem 5.7. Suppose $f \in \mathbb{R}[X]$ is homogeneous, say $f(X)=\sum_{|\alpha|=d} a_{\alpha} X^{\alpha}=$ $\sum_{|\alpha|=d} c(\alpha) b_{\alpha} X^{\alpha} \in \mathbb{R}[X]$, where $c(\alpha):=\frac{d !}{\alpha_{1} ! \cdots \alpha_{n} !}$. Let $L=L(f):=\max _{|\alpha|=d}\left|b_{\alpha}\right|$ and $\lambda=\lambda(f):=\min _{X \in \Delta_{n}} f(X)$. Then for

$$
N>\frac{d(d-1)}{2} \frac{L}{\lambda}-d,
$$

$\left(x_{1}+\cdots+x_{n}\right)^{N} f\left(x_{1}, \ldots, x_{n}\right)$ has positive coefficients.

Substituting this theorem for Corollary 3.2, we obtain our results. The proof of this theorem is contained in the forthcoming paper [19].

\section{REFERENCES}

1. S. Bernstein, Sur la représentation des polynômes positifs, Soobshch. Kharkov matem. ob-va, ser. 2, 14 (1915), 227-228.

2. P. Borwein and T. Erdélyi, Polynomials and polynomial inequalities, GTM 161, SpringerVerlag, New York, 1995. MR 97e:41001

3. R. A. DeVore and G. G. Lorentz, Constructive Approximation, Springer-Verlag, Berlin, 1993. MR 95f:41001

4. T. Erdélyi, Estimates for the Lorentz degree of polynomials, J. Approx. Theory 67 (1991), 187-198. MR 92m:41023

5. T. Erdélyi and J. Szabados, On polynomials with positive coefficients, J. Approx. Theory $\mathbf{5 4}$ (1988), 107-122. MR 91g:41026

6. J. Franel, solution, Intermèd. des math. 1 (1894), 253-254.

7. E. Goursat, solution, Intermèd. des math. 1 (1894), 251.

8. D. Handelman, Representing polynomials by positive linear functions on compact convex polyhedra, Pac. J. Math. 132 (1988), 35-62. MR 90e:52005

9. G. H. Hardy, J. E. Littlewood, and G. Pólya, Inequalities, 2nd ed., Cambridge Univ. Press, 1952. MR 13:727e

10. F. Hausdorff, Summationsmethoden und Momentfolgen I, Math. Zeit. 9 (1921), 74-109.

11. C. Hermite, problem, Intermèd. des math. 1 (1894), 65-66.

12. S. Karlin and L. S. Shapley, Geometry of Moment Spaces, Memoirs of the Amer. Math. Soc, 12, 1953. MR 15:512c

13. S. Karlin and W. J. Studden, Tchebycheff systems: with applications in analysis and statistics, Interscience, New York, 1966. MR 34:4757 
14. J. A. de Loera and F. Santos, An effective version of Pólya's theorem on positive definite forms, J. Pure Appl. Alg. 108 (1996), 231-240. MR 97b:12001

15. J. A. de Loera and F. Santos, Correction to An effective version of Pòlya theorem on positive definite forms, J. Pure Appl. Alg., to appear.

16. C. A. Micchelli and A. Pinkus, Some remarks on nonnegative polynomials on polyhedra, in Probability, Statistics and Mathematics: Papers in honor of Samuel Karlin (T. W. Anderson, et al. eds.), Academic Press, Boston, 1989, pp. 163-186. MR 91h:26014

17. G. Pólya, Über positive Darstellung von Polynomen Vierteljschr, Naturforsch. Ges. Zürich 73 (1928 141-145, in Collected Papers 2 (1974), MIT Press, 309-313.

18. G. Pólya and G. Szegö, Problems and Theorems in Analysis II, Springer-Verlag, New York, 1976.

19. V. Powers and B. Reznick, A new bound for Pólya's Theorem with applications to polynomials positive on polyhedra, to appear in Proceedings of the MEGA 2000 conference.

20. B. Reznick, Some Concrete Aspects of Hilbert's 17th Problem, to appear in RAGOS Proceedings, Contemp. Math. 253 (2000), 251-272.

21. J. Sadier, solution, Intermèd. des math. 1 (1894), 251-253.

22. C. Scheiderer, Sums of squares of regular functions on real algebraic varieties, to appear in Trans. Amer. Math. Soc. CMP 99:09

23. K. Schmüdgen, The K-moment problem for compact semi-algebraic sets, Math. Ann. 289 (1991), 203-206. MR 92b:44011

24. G. Stengle, Complexity estimates for the Schm̈udgen Positivstellensatz, J. Complexity 12 (1996), 167-174. MR 97d:14080

Department of Mathematics, Emory University, Atlanta, Georgia 30322

E-mail address: vicki@mathcs.emory.edu

Department of Mathematics, University of Illinois at Urbana-Champaign, Urbana, ILLINOIS

E-mail address: reznick@math.uiuc.edu 